\title{
Research on the Failure of Brittle Rock-like Samples Based on Its Acoustic Emission Characteristics under the Condition of Uniaxial Compression
}

\author{
Zemin Zeng ${ }^{1, a}$, BinWang ${ }^{1,2,3,4, b}$, Mengju Zhang ${ }^{1, c}$
}

${ }^{1}$ School of Energy and Safety Engineering, Hunan University of Science and Technology, Xiangtan

${ }^{2}$ State Key Laboratory for Geomechanics and Deep Underground Engineering, China University of Mining and Technology,Beijing,100083,China

${ }^{3}$ Mining Engineering Postdoctoral Scientific Research Station, Hunan University of Science and Technology, Xiangtan, Hunan 411201, China

${ }^{4}$ Hunan Provincial Key Laboratory of Safe Mining Techniques of Coal Mines, Xiangtan, Hunan 411201, China

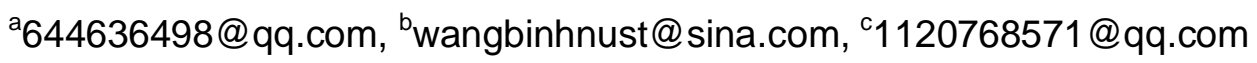

Keywords: Brittle Rock-like Samples, Acoustic Emission Characteristics, Uniaxial Compression, the Starting Time of Failure

ABSTRACT: There is less research on the accordance relationship between AE characteristics and failure process including the starting time and duration of failure of brittle rock. The different brittleness rock-like samples made of rosin and grout mortar have been researched by the uniaxial compression test and the acoustic emission (AE) experiments. Compared with the samples without rosin, brittleness index of the rock-like samples with rosin increase by $30 \%-40 \%$, starting time of failure is $41 \%-57 \%$ later and failure duration is $38 \%-48 \%$ shorter than those with lower brittleness. Combined with the initial failure time of rock-like samples and its ringdown count rate, failure characteristics among those rock-like samples with different brittleness are analyzed. Seen from the $\mathrm{AE}$ ringdown-time curves, there exists a stable period until the $\mathrm{AE}$ ringdown rate reaching to peak point, the length of its duration is directly proportional to the brittleness of rock-like samples, and the longer the duration is, the more energy can be accumulated, meanwhile the failure velocity is faster than those rock-like samples with lower brittleness. All these results can offer some references to research the starting time of failure and AE features of brittle rock and effectively promote the prediction of failure of brittleness rock.

\section{Introduction}

With the rapid development of deep resources exploitation and underground engineering, some geological disasters are easily induced when hard-brittle rocks are buried in high stress, for example, rockburst seriously threat the stability of tunnels and chamber structures[1], so hard-brittle rocks have been researched from different angles, such as its brittleness descriptions, its index evaluations, failure mechanisms. It's a fact that there exist AE phenomena during the process of rock failure in which the initiation, extension and rupture of micro-crack also appear, what's more, through researching and analyzing $\mathrm{AE}$ signals of rock, the changes of its inner structure can be deduced and its failure mechanism can be inversed. Therefore, rock $\mathrm{AE}$ technique, as a way of reflecting deformation and failure process of rock, has been widely used in the mechanism study of rock during damaging stress process[2,3]. Sun Qiang[4]conducted the AE experiment under uniaxial loading of sandstone to recognize different feature points among the deformation stage. Based on EMD, Welch spectrum and BP neural network method, Liu JianWei[5] extracted and recognized acoustic parameters, and then analyzed the time frequency features of three kinds of brittle rock. $\mathrm{Wu}$ XianZhen[6] studied the differences of mechanism property and sequence time of acoustic emission domain characteristics and sequence fractal characteristics of different rocks. At present, for lack of knowledge about the starting failure time of rock, accurate distinguishing basis and 
theory foundation, and too much dependence on experiential judgments of practical monitoring, result in the lower failure forecast rate in monitoring[7]. Brittle rock, which has higher ratio of compression to tension and energy resilience, are composed of multiple particles substance, its failure is mainly controlled by inner micro-cracks. Above all, these factors make it harder to predict the failure of rock. Consequently, It's vital to study AE features during the process of failure in which rocks are under compression. Furthermore, it's significant to reveal the relationship between failure process and its $\mathrm{AE}$ features of rock-like samples, and to further understand the failure mechanism which can help to provide reasonable failure precursory criterions of brittle rock. On the foundation of the previous related study and with the combination of the stress variation characteristics and AE monitoring signals of two groups of different brattle rock-like samples, this paper will study the process and mechanisms of deformation and failure of brattle rocks, to figure out an accurate starting failure time and velocity of crack propagation.

\section{Experiment Preparation of Samples and Process}

Similarity Materials' Composition and Proportion. Aiming at AE monitoring under the uniaxial compression of two groups of rock-like samples with differ brittleness, the physical and mechanical properties are required to be same between similarity samples and original rock. Li DongPing[8] studied the failure mode of mixed materials composed by cement and gypsum through laboratory test, and found out that the failure features of this materials were similar to brittle rocks, whose failure process include elastic stage, elastic-plastic stage and the final failure period. At the same time, cement as a cementing material can bond granular fine sands into molding material. In addition, this compound materials have cheaper raw material and easy manufacture process on simulating rock-like samples. The mix proportion plan is made out by analyzing previous researches[9,10], the similar simulation material is constituted by PO32.5 ordinary Portland cement, fluvial sands, water and building gypsum, according to the proportion of 1:5.3:1.8:2.7. In addition, in order to improve fluidity of the mortar concrete mixing material, water reducing agent is added into this compound material. As a fact that rosin serves as a brittle additive which can change plastic rock-like material into brittle one[11]. Preparation of two kind of different brittle rock-like samples by means of adding rosin or not in this experiment.

Preparation of Rock-like Samples. Customized cast iron moulds are used to process the rocklike cylinder samples whose height is $100 \mathrm{~mm}$ and whose diameter is $50 \mathrm{~mm}$. The raw materials are weighed and mixed according to the proportion above. Next, stirred the cement mortar into the state of viscous by using JJ-5 cement mortar agitator. Then, filled the mixed material into the cast iron mould and vibrated the mortar into uniform state on shaking table. Twelve hours later, cement mortar form into a preliminary stable state. Finally, dismantled the mould. The primary rock-like samples are prepared. Before gaining the required standard samples, 28 days maintain in constant temperature and humidity environment are vital.

Uniaxial Compression and AE Experiment. The experiments are conducted by the RMT-150C rock mechanics electron servo control test system. The rock-like samples are uniaxially compressed with the speed of $0.005 \mathrm{~mm} / \mathrm{s}$. In order to decrease the end-effect of samples during test, emery papers are used to polish up the top and bottom surface of samples where lubricants would be added after being polished up. AEwin testing system are used during the experiment. The signals of AE are collected by double channel. There is a layer of thin and uniform coupling medium which is smeared on the interface where AE probe and sample contact with each other. To check whether they contact with each other well or not, the breaking lead test is used near the interface. Firstly, placed the prepared samples on pressure platform of tester. Secondly, adjusted the receiving threshold value of AE. Finally, carried out the uniaxial compression test and the AE experiment. At the same time, recorded and shot the process of initiation, extending and transfixion of cracks clearly and completely. 


\section{Results and Analysis}

Brittleness Index Evaluation and Mechanical Properties of Rock-like Samples. To study the relationship between $\mathrm{AE}$ features and failure process on the uniaxial compression based on different brittleness rock, it's important to evaluate the brittleness of those prepared samples. By introducing peak strain and strength, and analyzing geometric condition of stress-strain curve on the whole deformation and failure phrase, the brittleness expression of soft and damage rocks under the condition of triaxial compression can be obtained[12]. It is:

$$
m=\frac{1}{\ln \left\{E \varepsilon_{\text {peak }} /\left[\sigma_{\text {peak }}-\mu\left(\sigma_{2}+\sigma_{3}\right)\right]\right\}}
$$

Where $m$ is the brittleness evaluation index, and $E$ is elastic modulus, $\varepsilon_{p \in a k}$ is peak strain and $\sigma_{\text {peak }}$ is peak stress, $\mu$ is Poisson ratio, $\sigma_{2} 、 \sigma_{3}$ is the axial stress and confining pressure. Due to the situation in this paper is uniaxial compression, so $\sigma_{2}, \sigma_{3}$ is both 0 , the expression in uniaxial compression is:

$$
m=\frac{1}{\ln \left(E \varepsilon_{\text {peak }} / \sigma_{\text {peak }}\right)}
$$

Table 1 Uniaxial compression test results and brittleness index evaluation table

\begin{tabular}{ccccc}
\hline Parameters & $\begin{array}{c}\text { Elastic } \\
\text { modulus }\end{array}$ & $\begin{array}{c}\text { peak stress } \\
\sigma_{\text {peak }}(\mathbf{M P a})\end{array}$ & $\begin{array}{c}\text { peak strain } \\
\varepsilon_{\text {peak }}\end{array}$ & $\boldsymbol{m}$ \\
\hline P-A $\mathbf{A}_{\mathbf{1}}$ & 1.09 & 3.74 & 5.84 & 2.06 \\
$\mathbf{P}-\mathbf{A}_{2}$ & 1.06 & 4.03 & 5.93 & 2.27 \\
$\mathbf{P}-\mathbf{A}_{3}$ & 0.97 & 3.62 & 6.49 & 1.82 \\
$\mathbf{S}_{\mathbf{A}}$ & 0.68 & 4.60 & 6.69 & 2.78 \\
$\mathbf{S}_{\mathbf{1}}$ & 0.55 & 4.16 & 10.55 & 2.97 \\
$\mathbf{S}_{\mathbf{2}}$ & 0.59 & 4.60 & 11.04 & 2.87 \\
\hline
\end{tabular}

Shown as the table, the $m$-value of samples with rosin is bigger than those without rosin, which shows that the brittleness of the samples with rosin is higher. This result accords with that of previous research[13].

By analyzing the process of stress variations under the uniaxial compression and comparing the features of ringing counting rate, some differences can be figured out between those two groups of rock-like samples with different brittleness. As for the failure test of rock-like samples with lower brittleness, the AE signals are weak and the variation range is narrow. With the increasing of load, the peak of $\mathrm{AE}$ ringdown rate have an obvious variation. $\mathrm{AE}$ ringdown rate of $\mathrm{P}-\mathrm{A}_{1}$ sample increase obviously when samples are loaded to the $69.2 \%$ peak loading, and the failure develop from elastic period into unsteady failure development stage and finally extend to development stage after failure. The whole sustainable time of failure is at $60 \mathrm{~s}$, and then the $\mathrm{AE}$ rate fluctuates in 50 times per second. AE ringing count rate of $\mathrm{P}-\mathrm{A}_{2}$ sample reaches to the maximum when loaded to the $71.5 \%$ total loading. And from that time the variation range is relatively wide which keeps about $61 \mathrm{~s}$. After that the $\mathrm{AE}$ rate begins to decrease and the variation range of acoustic emission is narrow until the whole loading to end. The peak load of $\mathrm{P}-\mathrm{A}_{3}$ sample appears at the $69.6 \%$ peak loading, and then the failure develops into unsteady failure development stage. The obvious AE fluctuate period is from 94 s to $148 \mathrm{~s}$. According to the features of $\mathrm{AE}$ in this group of samples, there is a stable period of $\mathrm{AE}$ signal before $\mathrm{AE}$ ringdown count rate having a sudden increasing, which is about 9s-12s.

As for the samples with higher brittleness which add rosin, its AE ringdown rate changes obviously and lasts about 120s-150s, and after that it decreases to a lower degree and fluctuated in a narrow range. On the one hand, because rosin plays as foaming agent it results in some smaller vesicles, which make the particles distribute unevenly. For this reason the particles with vesicles strength can be crushed early, so its AE appeared from the starting of the high brittle samples. At the same time, rosin has the same function as binder, which increases the mutual connection between inner structures after the weaker particles being crushed. Therefore, the viscosity between 
particles increased and the samples can't be crushed. Then the failure develop into elastic deformation stage in which the AE ringdown rate hardly fluctuate. This period means the stable stage for the high brittle rocks which kept about 21s-29s. The failure duration of high brittle sample is twice as long as the lower brittle samples'. Moreover, the AE ringdown rate grows suddenly to 91\%-95\% of peak load, and its violent vibrate of AE last about 35s-38s. Compared with the samples with lower brittleness, its time is shortened nearly by half .

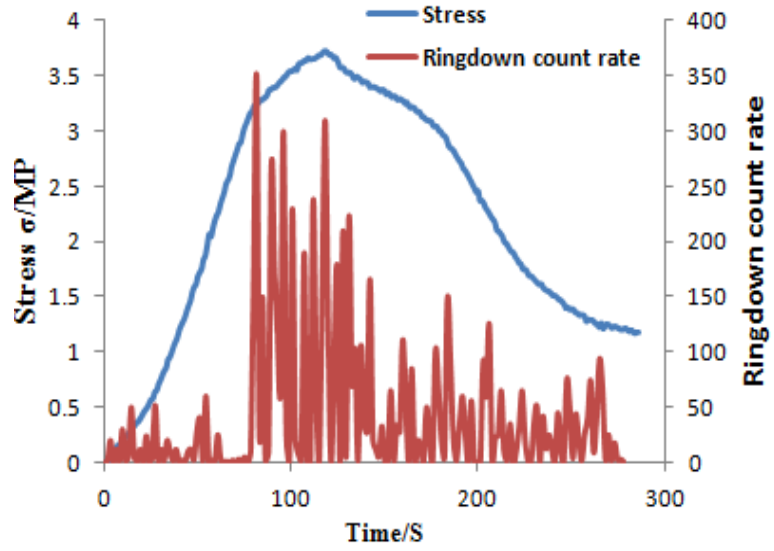

(a) Stress-Time-Ringdown count rate curve of $\mathrm{P}-\mathrm{A}_{1}$

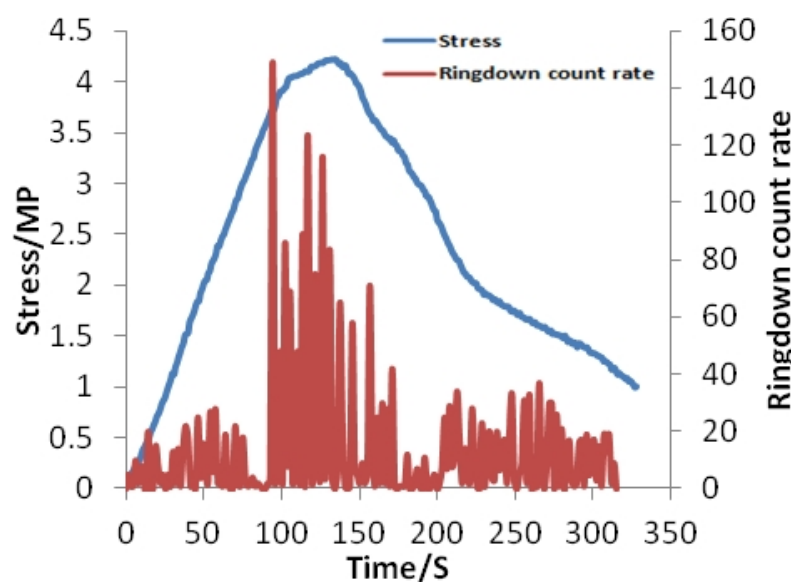

(c) Stress-Time-Ringdown count rate curve of $\mathrm{P}-\mathrm{A}_{3}$

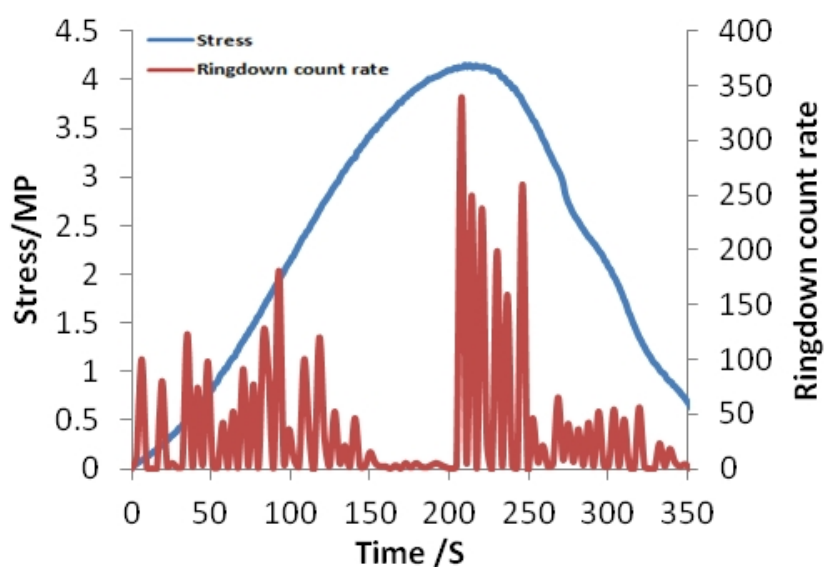

(e) Stress-Time-Ringdown count rate curve of S-A

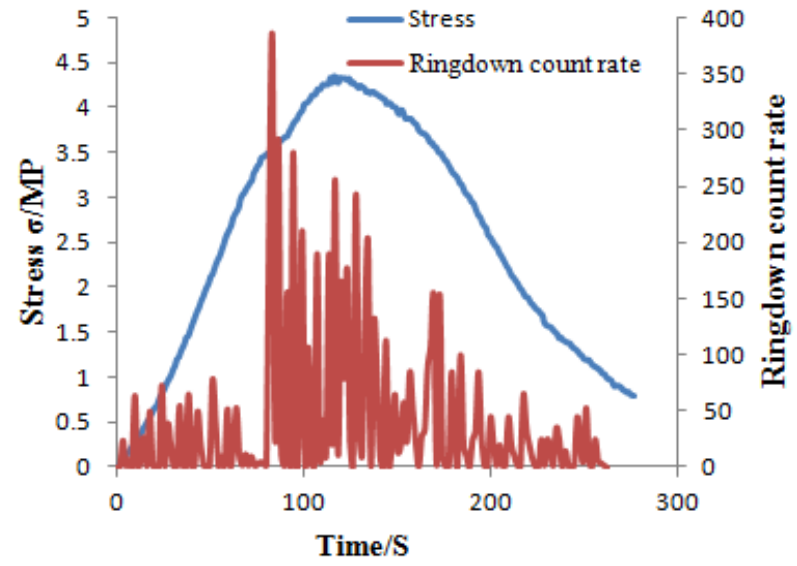

(b) Stress-Time-Ringdown count rate curve of $\mathrm{P}-\mathrm{A}_{2}$

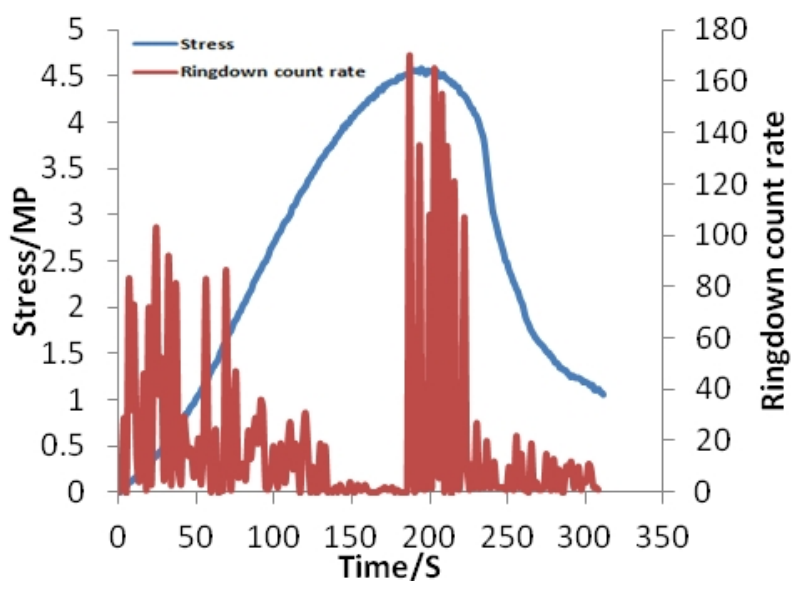

(d) Stress-Time-Ringdown count rate curve of S-A

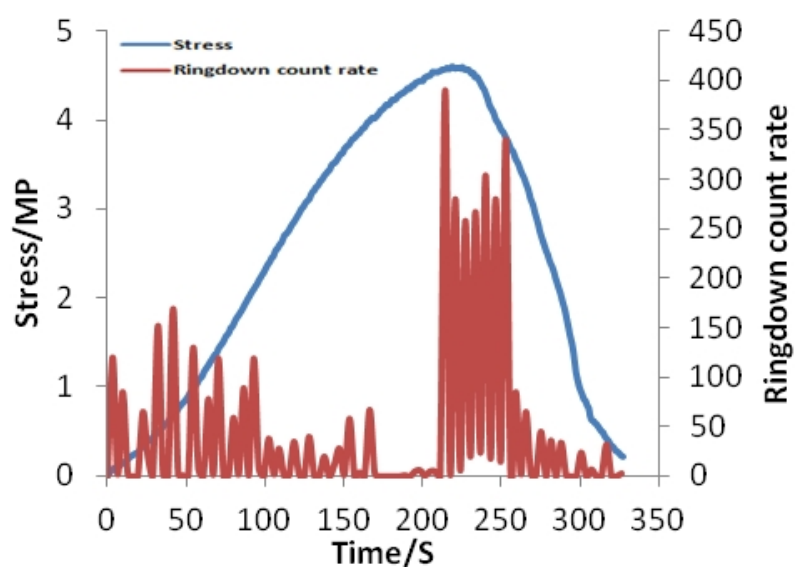

(f) Stress-Time-Ringdown count rate curve of S- $\mathrm{A}_{3}$

Fig.1. AE features of samples during the process of compression

With rosin added into samples, brittleness and compressive strength of rock-like samples can be both increased, which needed more time before samples being crushed. With loading increased gradually, the elastic strain energy in inner of samples increase simultaneously, the longer time of compression is, the more energy is accumulated in samples under the same loading velocity. While 
AE signal during this period is slightly, so the stable stage of two groups is different. The stable stage of high brittle rock-like samples is two times longer than the lower one. With the compression increasing continually, the accumulated elastic energy can't meet the need that used for microcracks to develop firmly. This will lead a qualitative change of micro-cracks in which the elastic energy releases violently and crack increases and extends rapidly, so the AE ringdown rate changes dramatically.

\section{Differences between Rock-likes Samples with Differ B rittleness in Failure Process.}

1)Combining the experimental observation during test with the video and photographs, the test shows that the starting failure time is different for those two groups of rock-like samples. From table 2, it is clear that the starting failure time of lower brittleness samples advances by $41 \%-57 \%$ compared with higher brittle samples.

2)The failure of rock-like samples with higher brittleness is $38 \%-48 \%$ shorter than those with lower brittleness. And the velocity of cracks' developing and failure are faster of the higher brittleness samples during the whole rupture process. And there are some corresponding relationships between starting failure time and the rate of cracks failure. As cracks appear relatively late and compression increased continually, the elastic energy inside samples increase simultaneously. When cracks appear suddenly, the energy inside samples release sharply, so the failure rate is faster of those samples with higher brittle.

3)The interval time $(\Delta T)$ between the time of peak-value point of $\mathrm{AE}$ ringdown rate and appearance of macroscopic cracks is different. Seen from table 1 and table 2, brittleness index of P$\mathrm{A}_{3}$ sample is minimum, its $\Delta T$ is $33 \mathrm{~s}$, while $\mathrm{S}-\mathrm{A}_{3}$ sample is maximum its $\Delta T$ is $6 \mathrm{~s}$, which means that when $\mathrm{AE}$ ringdown rate reach to the maximum samples with higher brittleness begin to damage. The higher the brittleness is , the shorter the $\Delta T$ is.

Table 2 Failure features of rock-like samples during unaxial compression

\begin{tabular}{cccc}
\hline $\begin{array}{c}\text { Number of } \\
\text { samples }\end{array}$ & $\begin{array}{c}\text { Starting time section of } \\
\text { macroscopic failure/s }\end{array}$ & $\begin{array}{c}\text { Peak-value time point of } \\
\text { AE ringdown rate/s }\end{array}$ & $\begin{array}{c}\text { Duration of failure } \\
\text { from start to steady/s }\end{array}$ \\
\hline $\mathbf{P}_{\mathbf{A}} \mathbf{A}_{\mathbf{1}}$ & $97-100$ & 81 & $183-186$ \\
$\mathbf{P}_{\mathbf{A}}$ & $102-105$ & 83 & $173-180$ \\
$\mathbf{P}_{\mathbf{A}}$ & $115-118$ & 85 & $205-208$ \\
$\mathbf{S}_{\mathbf{3}}$ & $196-199$ & 187 & $114-117$ \\
$\mathbf{S}_{\mathbf{1}}$ & $209-212$ & 208 & $115-118$ \\
$\mathbf{S}_{\mathbf{2}}$ & $223-225$ & 214 & $107-110$ \\
\hline
\end{tabular}

\section{Conclusions}

(1)As for brittle rock material, there is a stable stage until the AE ringdown rate reaching to peak under uniaxial compression load, its duration of stable stage is closely related to the brittleness of rock-like samples. The higher the brittleness is, the longer the stable stage will be, and the more energy will be accumulated. Therefore, the failure time of rock can be judged by the stable stage before $\mathrm{AE}$ ringdown reaching to peak in engineering practice.

(2)Rosin can increase the uniaxial compressive strength and also improve the brittleness of rocklike samples in the similar simulation test of rock, which provides reference significance for preparing rock-like samples with higher brittle whose starting time of failure is later than those with lower brittleness, while, the duration of failure is smaller and the velocity of failure is faster.

(3)According to the analyses of the features of $\mathrm{AE}$ signals and the starting time of failure of the brittle rocks, this paper has enhanced the understanding about the failure process for brittle rock and has provided some significant references for geologic disasters which are induced by the failure of brittle rock. 


\section{Acknowledgements}

This work is financially supported by the Scientific Research Fund of Hunan Provincial Science and Technology Department (No.2013TP4057-2), State Key Laboratory for GeoMechanics and Deep Underground Engineering, China University of Mining \& Technology (No. SKLGDUEK1214), National Natural Science Foundation of China (No.51474103).

\section{References}

[1] Zhang Jingjian, FU Bingjun. Rockburst and its criteria and control. Chinese Journal of Rock Mechanics and Engineering 2008;27(10):2034-2042(in Chinese).

[2] Xu Ze-min, HUANG Run-qiu, FAN Zhu-guo. Progress in research on rock burst hazard of long tunnel with large section. Journal of Natural Disasters 2004;13(2):16-23(in Chinese).

[3] Dai S T, Labuz J F. Damage and failure analysis of brittle materials by acoustic emission, Rock Mechanics and Rock Engineering 2005;38(1):1-19.

[4] Ganne P, Vervoort A, Wevess M. Quantification of pre-break brittle damage correlation between acoustic emission and observed micro-fracture, International Journal of Mechanics \& Mining Sciences 2007,44(5):720-729.

[5] Sun Qiang, Lei Xue. Research on AE variation of sandstone brittle failure with along loading by uniaxial compression, Chinese Journal of Solid Mechanics 2012;33(5):541-547(in Chinese).

[6] Liu Jian-wei1, Wu Xian-zhen, Liu Xiang-xin, Yu Yuan-yuan, Hu Wei, Yin Li-bing. Timefrequency characteristic and signal recognition of acoustic emission generated from different rock brittle failure, Nonferrous Metals Science and Engineering 2013;(496):73-77(in Chinese).

[7] Wu Xian-zhen, Liu Xiang-xin, Liang Zheng-zhao, You Xun, Yu Min, Experimental study of fractal dimension of AE serials of different rocks under uniaxial compression 2012;27(2):224227(in Chinese).

[8] Li Shulin, Yin Xiangang, Wang Yongjia, Fang Haiyan. Study on acoustic emission characteristics of uniaxial compressive rock failure. Chinese Journal of Rock Mechanics and Engineering 2006;27(2):224-227(in Chinese).

[9] Li Dong-ping, Wang Qian-yuan, Zhang Zeng-xiang, Wei Wei, LI Zheng. Similar test research on property of simulated rock. Journal of Hebei University of Engineering (Natural Science Edition) 2007;24(2):12-15(in Chinese).

[10] Li Liangui ,Xu Wensheng ,X u Yingnian, Wang Yuanhan. Experimental study on simulation materials of rockburst. J .Huazhong Univ .of Sci.\& Tech. 2001;29(6) :80-82(in Chinese).

[11] Li Shucai, Yang Lei, Li Mingtian, Zhang Ning. Influences of 3D internal crack dip angle on tensile mechanical properties and fracture features of rock-like material. Chinese Journal of Rock Mechanics and Engineering 2009;28(2) :281-289(in Chinese).

[12] Yang Ming-hui, Zhao Ming-hua, Cao Wen-gui. Method for determining the parameters of statistical damage softening constitutive model for rock. SHUILI XUEBAO 2005;36(3):345349(in Chinese).

[13] Wang Shenzu Brittle-ductile transition and plastic-flow networks in rocks. Progress in Geophysics 1993;8(4):25-37(in Chinese). 